\title{
Fostering multidisciplinary collaborations
}

\author{
We discuss the role of computational science as a multidisciplinary field and our editorial practices to promote \\ communication and research across different disciplines.
}

$T^{1}$ he computational science community continues to astonish us with scientific discoveries that have the potential to solve the most challenging problems in our society. To highlight a recent advancement, protein-structure prediction models using deep learning techniques ${ }^{1}$ achieved impressive levels of accuracy and efficiency, which can further aid in the design of new drugs and enzymes. As another example, a year and a half after the start of the coronavirus disease 2019 (COVID-19) pandemic, by using sophisticated computer simulations, scientists now have a clearer understanding of the whole life cycle of the severe acute respiratory syndrome coronavirus 2 (SARS-CoV-2) virus when it infects human cells, which can provide valuable insights for developing strategies to cease the spread of the virus ${ }^{2}$. The emergent quantum computing area is also making headlines across various fields - such as chemistry $^{3}$, biolog $\mathrm{y}^{4,5}$, climate science ${ }^{6}$ and machine learning ${ }^{7}$ - in an effort to develop new quantum algorithms to solve longexisting problems that cannot be properly addressed on classical computers.

It is worth noting that all of the aforementioned examples have one aspect in common: they are all a product of close collaborations between researchers from different fields. The protein-structure prediction development, for instance, would not have been possible without strong collaborations between the machine learning, bioinformatics, microbiology and chemical biology communities. Similarly, combined advances from computational biology, virology, epidemiology and computational visualization have furthered our understanding of the SARS-CoV-2 virus. It also goes without saying that quantum computing calls for multidisciplinary collaboration, involving a multitude of fields, including physics, computer science, chemistry, biology, and so forth.

This collaboration aspect is not necessarily surprising. As a matter of fact, it is impossible to find computational science research that is not multidisciplinary. The main goal of this field is to take state-of-the-art methods and models from computer and mathematical sciences (for instance, machine learning algorithms, graph theory and optimization strategies) and apply them to other disciplines: computational science is inherently multidisciplinary. And because multiple disciplines can use the same or similar computational techniques, this fosters communication between different areas: an approach developed in one field has the potential to be repurposed and reused in another field. Therefore, we argue that computational science bolsters existing communication channels and further creates new ones between researchers across different fields in order to solve our most challenging, albeit exciting, research problems.

At Nature Computational Science, it is one of our main goals to promote the role of computational science in fostering multidisciplinary research. Looking at the Aims \& Scope page of our journal, our readers might be impressed, and at the same time puzzled, by the many different disciplines that are under our scope. However, this 'ambitious' list reflects nothing more than the critical place where computational science stands nowadays: we want to celebrate the importance of computational science in a large number of fields. We also take into consideration the potential interest of a paper to a wide range of disciplines when deciding on whether to send it out to peer review ${ }^{8}$. Finally, our non-primary research content (for instance, Reviews, Perspectives and Comments) is mainly dedicated to covering topics that are of interest to a broad range of researchers.

While multidisciplinary research is very beneficial to the research community, we also realize that there are barriers for scientists to communicate across different domains ${ }^{9}$, including the lack of mutual understanding in outcome expectations, the differences in technical vocabulary and the distinct communication cultures. In the context of our editorial process, we make an effort to tackle these challenges in different ways. For instance, in the peer review process, we always aim to invite researchers with varied expertise to make sure that we have taken into account multiple perspectives of a paper. If a paper is accepted for publication, we perform careful editing to clarify any jargon or scientific terms that might be obscure to our multidisciplinary readership; if it is still difficult for the paper to be comprehended by non-experts, we have the option to invite a renowned researcher to write a News \& Views piece to accompany that paper, where the novelty of the paper can be better described and disseminated to our readers. We are, of course, always open to suggestions from our readers, authors and referees on how to improve our editorial process to ensure that our papers can reach broader audiences.

As new computing architectures are developed (for instance, exascale, quantum, optical and neuromorphic computing) and as more data become available, the computational science field will certainly continue to progress at a faster pace. Strong multidisciplinary collaboration will be vital for addressing our society's most urgent challenges and for enabling our greatest future achievements.

Published online: 20 August 2021 https://doi.org/10.1038/s43588-021-00123-x

References

1. Jumper, J. et al. Nature https://doi.org/10.1038/s41586-021-03819-2 (2021).

2. Scudellari, M. Nature 595, 640-644 (2021).

3. McArdle, S., Endo, S., Aspuru-Guzik, A., Benjamin, S. C. \& Yuan, X. Rev. Mod. Phys. 92, 015003 (2020).

4. Fedorov, A. K. \& Gelfand, M. S. Nat. Comput. Sci. 1, 114-119 (2021).

5. Emani, P. S. et al. Nat. Methods 18, 701-709 (2021).

6. O'Brien, J. How quantum computing could beat climate change. World Economic Forum https://go.nature.com/3iodjcT (2019).

7. Abbas, A. et al. Nat. Comput. Sci. 1, 403-409 (2021).

8. Nat. Comput. Sci. 1, 241 (2021).

9. Brown, R. R., Deletic, A. \& Wong, T. H. F. Nature $\mathbf{5 2 5}$ 315-317 (2015) 